\title{
La participación electoral de 2009, 2014 y 2019 en Ecuador: diagnóstico por cantones en un país con voto obligatorio
}

\section{The electoral participation of 2009, 2014 and 2019 in Ecuador: diagnosis by cantons in a country with mandatory voting}

William Eduardo Jiménez Paredes

Recepción: 1 de septiembre 2019

Aprobación: 15 de noviembre

\section{Resumen}

La presente investigación intentará responder ¿Cuál es el diagnóstico de la participación electoral en las votaciones municipales del 2009, 2014 y 2019 en Ecuador? Para cumplir con este objetivo, el objeto de estudio lo constituyen los 221 municipios en 11 años, con enfoque comparativo, entre las elecciones 2009, 2014 y 2019, a través del porcentaje de participación electoral por cantones. Como resultados se destacan que la tendencia de participación varía entre el $60 \%$ al $90 \%$. Utilizando el marco teórico sobre la calidad de la democracia y la participación electoral, con Munck y Verkuilen, Barreda, Altman, Mainwaring, Pérez-Liñán, Levine y Molina, Franklin, Marsh y Lyons, Wagner, Johann y Kritzinger, Geissel y Jung, Hadjar y Beck, entre otros, se describirán estos resultados.

Palabras clave: Ecuador, democracia subnacional, sufragio cantonal, participación electoral.

\section{Abstract}

This research will try to answer: Which is the diagnosis of the electoral participation in the municipal votes of 2009, 2014 and 2019 in Ecuador? To accomplish this objective, the object of study is the 221 municipalities in 11 years, with a comparative approach, between the elections 2009, 2014 and 2019, through the percentage of electoral participation by cantons. As a result, the participation trend varies $60 \%$ to $90 \%$. By using the theoretical framework about the quality of democracy and electoral participation, with Munck and Verkuilen, Barreda, Altman, Mainwaring, Pérez-Liñán, Levine and Molina, Franklin, Marsh and Lyons, Wagner, Johann and Kritzinger, Geissel and Jung, Hadjar and Beck, among others, these outcomes are going to be described.

Key words: Ecuador, subnational democracy, cantonal suffrage, electoral participation. 


\section{Marco teórico}

\section{Democracia}

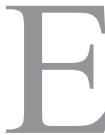

ste artículo inicia con dos conceptos de lo que es la democracia. Su noción "minimalista", por un lado, lo define Schumpeter (1975) como método democrático, entendido como "el arreglo institucional para llegar a decisiones políticas en el que los individuos adquieren poder para decidir por medio de una lucha competitiva por el voto de los ciudadanos" (O`Donnell, 2010: 24). Por otro lado, la democracia política moderna es un "sistema de gobierno en el que los gobernantes son responsables de sus acciones en el terreno público ante los ciudadanos, actuando indirectamente a través de la competencia y la cooperación de sus representantes electos" (Schmitter y Karl, 1996: 38).

En ese mismo orden de ideas, vale destacar cuáles son los procedimientos que hacen posible la democracia. Para ello se toman las siete condiciones de procedimiento mínimas, pero no suficientes, que deben estar presentes en las democracias políticas modernas, a lo que Robert Dahl (1991) denomina "poliarquía":

\section{Tabla 1.}

Condiciones presentes en democracias modernas

\begin{tabular}{|c|c|}
\hline 1. Funcionarios electos & El control de las decisiones corresponde a ellos. \\
\hline $\begin{array}{l}\text { 2. Elecciones libres e } \\
\text { imparciales }\end{array}$ & $\begin{array}{l}\text { Los funcionarios son elegidos mediante el voto en elecciones } \\
\text { limpias y en las cuales rara vez se emplea la coacción. }\end{array}$ \\
\hline 3. Sufragio inclusivo & Todos los adultos tienen derecho a votar. \\
\hline $\begin{array}{l}\text { 4. Derecho a ocupar } \\
\text { cargos públicos }\end{array}$ & $\begin{array}{l}\text { Todos los adultos tienen derecho a ocupar cargos públicos en el } \\
\text { gobierno. }\end{array}$ \\
\hline 5. Libertad de expresión & $\begin{array}{l}\text { La ciudadanía tiene derecho a expresarse, sin correr peligro de } \\
\text { sufrir castigos severos. }\end{array}$ \\
\hline $\begin{array}{l}\text { 6. Variedad de fuentes de } \\
\text { información }\end{array}$ & $\begin{array}{l}\text { La ciudadanía tiene derecho a probar diversas fuentes de } \\
\text { información, que no sólo existen sino que están protegidas por } \\
\text { la ley. }\end{array}$ \\
\hline 7. Autonomía asociativa & $\begin{array}{l}\text { La ciudadanía goza del derecho de constituir asociaciones } \\
\text { relativamente independientes, entre ellas partidos políticos y } \\
\text { grupos de intereses. }\end{array}$ \\
\hline
\end{tabular}

Fuente: Dahl (1991) y Schmitter y Karl (1996). 
Con este recorrido minimalista sobre la democracia, se considera oportuno entregar a las y los lectores, unas primeras reflexiones. Por un lado, se tienen retos sobre las metodologías para reducir posibles variables omitidas (Coppedge 2012: 326) respecto a la manera de analizar y estudiar la democracia a nivel nacional y subnacional. ${ }^{1}$

Por otro lado, se debe prestar mayor atención a las desigualdades geográficas en cada territorio durante los procesos políticos y de representación (McKay 2019: 2). Una hipótesis a indagar sería que, una democracia en una localidad pobre tendría mayor descontento y baja participación frente a otro lugar, que tiene mejores posibilidades socioeconómicas. Ante la poca posibilidad de cubrir las necesidades básicas de las personas, éstas podrían estar desanimadas a mantener una alta participación y control sobre sus respectivos gobernantes, puesto que su tiempo podría estar dedicado casi exclusivamente a llevar algún alimento básico a sus familias. Como lo sostiene Sen (2001: 79), el enfoque de capacidades va del capital humano, para generar ingresos, hacia una mayor integración de las personas, a través de la educación, entre otros factores, que afectan las auténticas libertades de la ciudadanía.

Con esta breve introducción se destaca el concepto que se utilizará como guía básica. Esta es la democracia representativa en tanto "incluye necesariamente un elemento de delegación: por medio de cierto procedimiento, una colectividad autoriza a ciertos individuos a hablar por ella" (O`Donnell 1992: 295). Este procedimiento recae en la participación electoral en un país.

\section{Calidad de la democracia y participación electoral}

En varias partes del mundo se ha investigado las transiciones hacia la democracia, su consolidación, o su calidad democrática. En el presente trabajo me enfocaré en la tercera opción, la cual ha suscitado debates respecto a su conceptualización, medición y agregación (Munck y Verkuilen 2002: 406).

1.- Ver Anexo 1, si el lector está interesado en conocer las debilidades y las fortalezas de las bases de datos disponibles sobre la democracia 
La calidad de la democracia no goza de un consenso elemental sobre su definición, a lo que podría existir desconcierto, por ejemplo, confundir calidad con nivel de democratización, pues la primera opción puede ser aplicada cuando ya existe un grado mínimo de la segunda (Barreda, 2013: 156). Para este artículo se utiliza el concepto de calidad en tanto permite medir a una buena democracia que le ofrece a la ciudadanía amplias libertades, igualdad política y control sobre políticas públicas (Diamond y Morlino 2004: 22).

Por tanto, se ha estudiado a la calidad de la democracia tomando ejes de la poliarquía de Dahl (1991). Altman y Pérez-Liñán (2002) utilizan las dimensiones sobre los derechos civiles, la participación y competencia efectivas; Mainwaring y Pérez-Liñán (2008) analizan sobre los derechos políticos y las libertades civiles. No obstante, otras investigaciones van más allá de la poliarquía: Diamond y Morlino (2004) presentan dimensiones que más adelante se las detallarán; mientras que Levine y Molina (2011) utilizan la decisión electoral, participación, accountability, responsiveness y soberanía (Barreda 2013: 160). Estos fueron algunos de los casos; por supuesto que existen otros más.

Tomando en cuenta que la calidad de la democracia no es un fenómeno de suma cero, sino una escala que va de lo mínimo aceptable a la situación óptima, autores como Levine y Molina se enfocan en los procedimientos y no en los resultados (Levine y Molina 2011: 97-99). De esa última idea se diferencian algunos autores, entre ellos, quienes presentan ocho dimensiones: Estado de derecho, participación, competencia, accountability vertical y horizontal, libertad, igualdad y responsiveness (Diamond y Morlino 2004: 22).

Debido a que el presente documento observará evidencia empírica sobre la participación, se considera oportuno reflexionar algunas pautas sobre este eje. La participación electoral puede ser vista como uno de los estados de salud que tiene una democracia (Wagner, et al. 2012: 373). La participación electoral tiende a disminuirse en varias partes del mundo, en Europa occidental lo analizaron Franklin, Marsh y Lyons (2004), o en América Latina, a través de estudios de percepción, se concluye que existe una crisis de representación y desconfianza de las instituciones por parte de la ciudadanía (Lagos 2018: 7). 
Una parte de la literatura afirma que los porcentajes de la participación electoral en países con voto obligatorio son más altas, que en donde este es voluntario (Morales y Reveco 2018: 2). Pero ¿qué medidas han tomado los actores políticos, donde el voto es voluntario? Algunos gobiernos europeos partieron de la idea que al incluir a personas de 16 años al sufragio, las tasas de participación aumentarían. Sin embargo, ¿estos jóvenes estaban motivados a usar este derecho político? ¿Con este tipo de medidas se mejora la calidad en cuanto a la participación? En Austria se evidenció que no tiene un impacto negativo en la legitimidad y en la calidad de las decisiones democráticas (Wagner, et al. 2012: 374-381).

La participación se suscribe en el debate académico dentro de la "legitimidad democrática de entrada", es decir, podrían ser un espejo de la voluntad de la gente y estar en sintonía con las preferencias de la comunidad (Scharpf 1999: 6). No obstante, esta participación puede ser relegada después de las elecciones. El sufragante ya no interactúa directamente con el Estado, sino hasta las próximas votaciones. Por supuesto que se puede apoyar la idea de Dalton (2009), en cuanto la participación electoral no es la única forma de crear vínculo democrático entre la ciudadanía y el sistema político.

Uno de estos nexos podría ser la revocatoria del mandato, leída desde un contexto actitudinal, como la insatisfacción de las personas contra un mandatario o contra la economía; o desde un contexto político, donde la oposición con mayoría en el concejo municipal o en el legislativo federal podría tener mayores incentivos para usar esta herramienta (Geissel y Jung 2018: 1360).

La participación puede ser analizada desde varios enfoques. Está el institucional, entendido como el estudio del sistema electoral, por ejemplo; también se ubica el aspecto socioeconómico y sociodemográfico de la ciudadanía; además, se encuentra el contexto en que se desenvuelven las personas, entendida como la generación política. Del primer enfoque pueden existir costos por no acercarse a votar según los niveles de competencia política; del segundo, pueden existir sesgos de clases sociales entre alta, media y baja para sufragar; y del tercero, pueden aparecer ciclos donde sube y baja la participación según los grupos de edad entre jóvenes y adultos (Morales y Reveco 2018: 5). 
Del mismo modo, la literatura presenta más formas de investigar a la participación electoral desde un diseño multinivel. En la fase micro están determinantes sociológicos, psicológicos o motivacionales; aquí también se incluye el interés por la política o la percepción de su eficacia. En el nivel macro se encuentran el régimen electoral y la calidad de las democracias (Hadjar y Beck 2010: 521).

La tasa de participación durante las elecciones en América Latina ha generado varias reacciones en la academia y en la política, con mayor fuerza durante el siglo XXI. Luna (2008) la denominó como paradójica, Castiglioni y Rovira (2016) como desconcertante. Esta disonancia se debe a las deficiencias en la representación política, los mecanismos democráticos y la accountability (Santana, et al. 2019: 153).

Lagos (2018) incluye otro elemento que podría influir en la disminución de la participación electoral: el deterioro de las élites en la región. Justamente, el voto que da a un grupo el acceso al poder no podría garantizar que la toma de las decisiones esté en función de los intereses de la sociedad. Por el contrario, durante el ejercicio del mandato, quien realmente toma las decisiones podrían ser grupos ajenos a las personas electas. Parafraseando a Acemoglu y Robinson (2012), el reparto del poder puede ser restrictivo y en beneficio de pocos actores.

Esta primera parte del documento inició con un breve recorrido sobre la democracia, enfatizando sobre la representativa, por la conexión que tiene con la participación electoral. Posteriormente, se definió la calidad de la democracia, entendida como una forma de medir a una buena democracia que le ofrece a la ciudadanía amplias libertades, igualdad política y control sobre políticas públicas (Diamond y Morlino 2004: 22). El objetivo de abordar la calidad democrática recae en que la participación electoral es una de las formas de observarla empíricamente a través de las elecciones seccionales del Ecuador. Con ello se da paso a la parte metodológica. 


\section{Metodología}

Para el presente documento se utilizará el enfoque teórico de Diamond y Morlino (2004) sobre cómo medir la calidad de la democracia. Esta propuesta se aplicará a gobiernos municipales del Ecuador del 2009 al 2019, según la pertinencia y accesibilidad a datos.

\section{Tabla 2.}

Dimensión de calidad democrática según Diamond y Morlino

\begin{tabular}{|c|}
\hline Dimensiones \\
\hline Estado de derecho \\
\hline Participación \\
\hline Competencia \\
\hline Accountability vertical \\
\hline Accountability horizontal \\
\hline Libertad \\
\hline Igualdad \\
\hline Responsiveness \\
\hline
\end{tabular}

Fuente: Diamond y Morlino, 2004: 23-28.

A continuación se describe qué significan cada una de estas dimensiones. El 'Estado de derecho' no sólo es el imperio efectivo de las normas jurídicas, también connota el principio de la supremacía de la ley. Respecto a la 'participación', esta es entendida como un conjunto de comportamientos, convencionales o no convencionales, legales o en el límite de la ley, que permite a la ciudadanía influir en el reclutamiento o en las decisiones de las autoridades políticas. Sobre 'competencia' cuando existe más de un actor involucrado en procesos de toma de decisiones políticas. Si hay competencia política, hay democracia, pero no necesariamente ocurre lo mismo si invertimos los términos, a esto se lo denomina democracias consensuales. Respecto al 'accountability vertical y horizontal', estas dan cuenta sobre la rendición de cuentas, la obligación que tienen las autoridades electas de responder por sus decisiones políticas cuando son demandados por la ciudadanía u otros órganos constitucionales (Morlino 2013:48). 
La 'libertad' está compuesta por derechos políticos, civiles y sociales. El primero incluye el derecho a votar, a competir por apoyo electoral, a ser electos en cargos públicos. De estos se desprenden otros más, por ejemplo cuando la ciudadanía puede ejercer influencia en candidatos. Los derechos civiles abarcan la libertad personal, la libertad de escoger el lugar de residencia, la libertad de pensamiento y de expresión, la libertad de asociación, entre otros. Los derechos sociales incluyen la salud, asistencia sanitaria y seguridad social, trabajo, medio ambiente sano, entre otros. La 'igualdad' tiene dos fases, primero se refiere a la igualdad ante la ley como la prohibición de discriminación por razones de sexo, religión, opiniones, etc. Y la segunda trata sobre la búsqueda de la igualdad sustancial, entendida como las barreras que limitan la igualdad social y económica (Morlino 2013:51).

'Responsiveness' trata la capacidad de respuesta del gobierno de satisfacer a los gobernados. No obstante, podrían existir dos límites, primero, las autoridades electas no siempre buscan entender las percepciones de la ciudadanía o responderlas, por el contrario los políticos podrían buscar influenciar sobre esas opiniones. Lo segundo, trata sobre los recursos que pueden tener las autoridades a su disposición para responder las necesidades de la población (Morlino 2013:55).

Después de revisar las dimensiones sobre la calidad democrática propuestas por Diamond y Morlino (2004), y tomando en cuenta la extensión del presente documento, se escogerá a la participación para analizarla en las elecciones seccionales del Ecuador. Dentro de esta se opta por sus oportunidades, entendidas como la intervención del electorado en las votaciones locales (Betancourt 2018: 41). Por supuesto que existen más variables por estudiar, pero esta fue la que se eligió, debido a la pertinencia y accesibilidad de datos. Por tanto, el objeto de estudio lo constituyen los 221 municipios en 11 años con enfoque comparativo, entre las elecciones 2009, 2014 y 2019, a través del porcentaje de participación electoral por cantones.

El estudio de las unidades subnacionales aporta a equilibrar el descuido que han tenido en el pasado (Giraudy, et al. 2019: 5), especialmente desde la Ciencia Política, siendo esta una contribución adicional del presente documento. Adicionalmente, su análisis constituye una herramienta 
indispensable para entender las tendencias políticas de la era contemporánea sobre las elecciones.

El documento trata sobre una investigación descriptiva dado que su propósito es detallar o especificar tendencias de la participación electoral a nivel cantonal. Este tipo de análisis son útiles para mostrar dimensiones de un fenómeno (Hernández, et al. 2010: 80). Adicionalmente, y tomando en cuenta que el análisis comparativo ocupa una de las posiciones centrales en la investigación de las ciencias sociales (Della Porta 2013:211), este trabajo académico pretende tener una perspectiva comparativa en el sentido de examinar patrones entre un número de casos, siendo un enfoque adecuado para explorar la diversidad (Ragin 2007: 181).

La pregunta de investigación es ¿cuál es el diagnóstico descriptivo de la participación electoral en las votaciones municipales del 2009, 2014 y 2019 en el Ecuador? Para responderla, este documento compara a las tres elecciones seccionales a través de los porcentajes de participación electoral obtenidos por cada cantón y para las 221 alcaldías.

La fórmula de cálculo se presenta a continuación, donde "P" es la participación electoral; "S" el número de sufragantes, léase personas que votaron; y la "E" como número de electores que constan en el padrón.

$$
\mathrm{P}=100 * \mathrm{~S} / \mathrm{E}
$$

La sistematización de los datos proviene de las bases de información que constan en la página web del Consejo Nacional Electoral (CNE), que son de acceso público y gratuito. Para las votaciones de 2009 y 2014 se extrajo del documento "Capítulo 6: indicadores electorales" que se ubica online en la sección estadísticas. Los insumos de 2019 fueron tomados de la misma dirección electrónica, de la sección: procesos electorales, elecciones seccionales, resultados. Aquí se ingresó al mapa interactivo para extraer la información por cada cantón. Posterior a ello, se limpió y se verificó las bases de datos en Excel para revisar errores de tipeo o de otra índole. 


\section{Resultados}

Se tomó a las oportunidades de la participación del electorado en las votaciones seccionales. Por supuesto que no es la única forma de observarlas, y que, como otras podrían ser debatibles. Por ejemplo, es más complejo medir para 221 cantones el acceso efectivo de las personas para acercarse a las urnas, especialmente en el sector rural; u observar empíricamente el sufragio voluntario de las personas.

La participación electoral en América Latina se encuentra por debajo de Europa occidental pero por encima de Estados Unidos entre 1990 al 2002 (PNUD 2004: 80). ${ }^{2}$ Tomando en cuenta que el voto es obligatorio en Ecuador, según el artículo 62 de la Constitución, y que la confianza ciudadana en las elecciones ha disminuido del $61,2 \%$ en 2004 al 49,3\% en 2016 (LAPOP 2018:16). A continuación se indican los resultados.

Del gráfico 1 se puede observar que durante los años analizados de las votaciones seccionales, la tendencia de participación varía entre el $60 \%$ al 90\% del padrón electoral en los 221 cantones del Ecuador. Como se puede observar, el porcentaje de participación entre cada elección, aumenta en su tendencia promedio.

Para los años 2009, 2014 y 2019, el cantón Déleg, de la provincia del Cañar, ocupa el último puesto donde, en promedio, 4 de cada 10 personas fueron a sufragar. Mientras que el cantón Isidro Ayora de la provincia del Guayas tiene la participación más alta, con un promedio de 9 de cada 10 personas quienes fueron a votar.

Si transformamos los porcentajes en números, se puede destacar que para 2019, el cantón con más participación tuvo 9.612 electores y sufragaron 9.195 personas, mientras que el cantón con el más bajo atributo analizado, tenía registrado en el padrón a 8.519 ciudadanos y ejercieron su voto 3.684 individuos.

2.- Para conocer el detalle de este tema ver Anexo 2. 
En principio, la participación electoral no tiene una fuerte relación directa con las condiciones de vida en Déleg e Isidro Ayora. No obstante, estos datos brindan, al lector y lectora, una fotografía de las necesidades básicas insatisfechas en que viven las personas en estos cantones, dejando una pista sobre los determinantes motivacionales de las personas respecto a las elecciones, parafraseando a Hadjar y Beck (2010). En Déleg, de 2.091 viviendas: 115 no cuentan con luz eléctrica procedente del servicio público; 1.208 no cuentan con servicio de recolección de basura, por tanto, la entierran o utilizan otras formas de desecharla; 1.735 no tienen servicio higiénico conectado a la red pública, es decir, desechan en letrina, entre otras maneras poco saludables; 662 no tienen acceso al agua por red pública, es decir usan este líquido vital del pozo, río o de la lluvia; y el 97\% no tienen disponibilidad de internet en sus hogares (INEC 2010).

Del mismo modo, en Isidro Ayora, de 2.959 viviendas: 384 no cuentan con luz eléctrica procedente del servicio público, 1.414 no cuentan con servicio de recolección de basura, por tanto, la entierran o utilizan otras formas de desecharla; 2.936 no tienen servicio higiénico conectado a la red pública, es decir, casi toda la población del cantón desecha en letrinas, entre otras maneras poco saludables; 2.269 no tienen acceso al agua por red pública, es decir usan este líquido vital del pozo, río o de la lluvia; y el 98\% no tienen disponibilidad de internet en sus hogares (INEC 2010). 
William EDUARdo JiMÉNEZ PAREDES

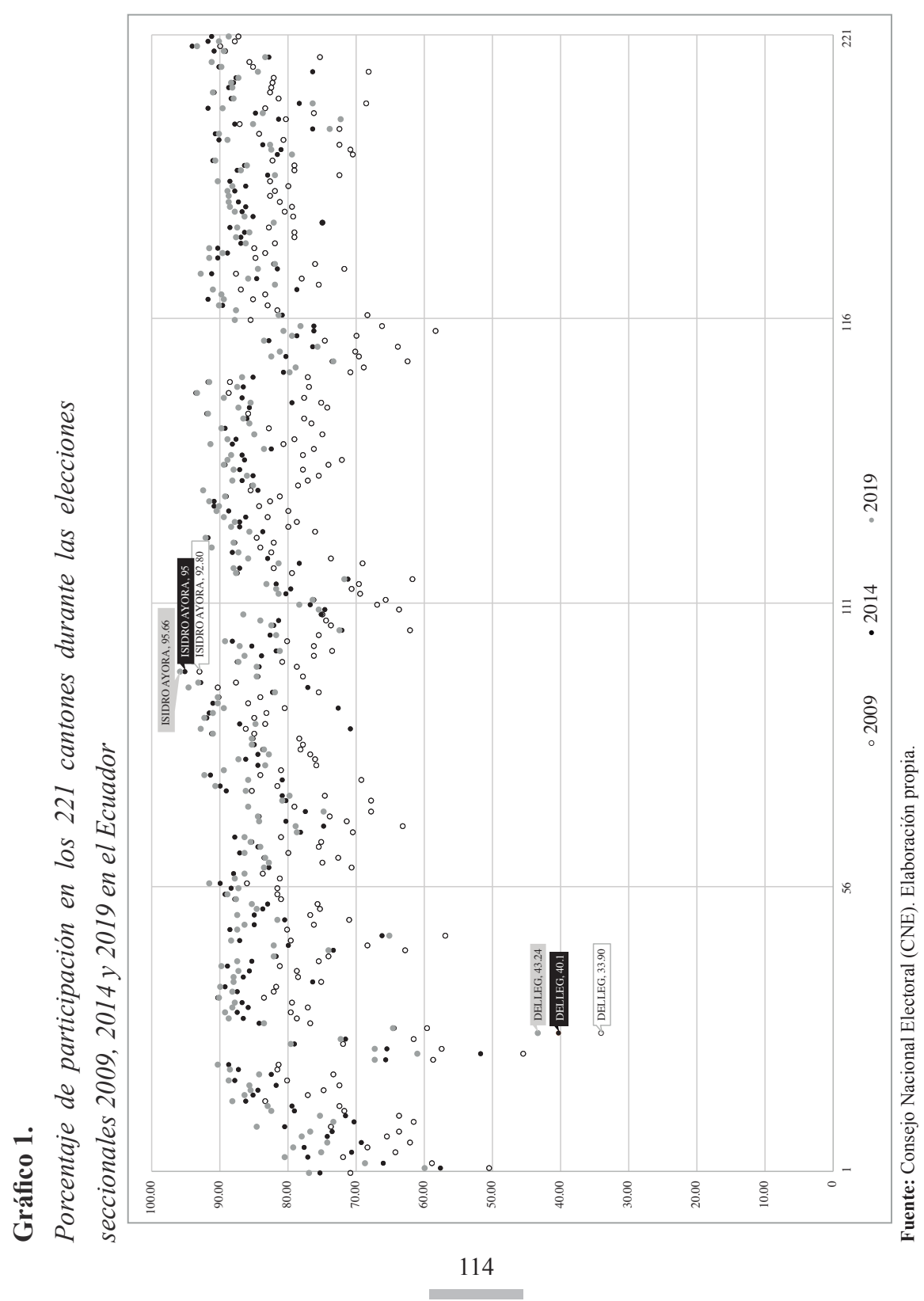


¿Qué factores explican la repetición en Déleg e Isidro Ayora como los cantones con menor y mayor participación para las tres elecciones locales? Esta interrogante es digna de investigarla con técnicas cualitativas y cuantitativas. No obstante, el presente documento tiene otros fines.

Por otra parte, como se observa en la tabla 3, únicamente en 18 de los 221 cantones, el porcentaje de participación ha disminuido entre 2009, 2014 o 2019. Ninguno de estos pertenece a la región Sierra. En las 203 unidades subnacionales restantes, la participación aumentó. Si se compara 2009 y 2019, arroja como resultado que dos ciudades han reducido este atributo de la calidad de la democracia, estos son Isabela y Santa Cruz. Es decir, la participación cayó en 1,90\% y 8,07\%, respectivamente.

Mientras que si observamos las elecciones de 2014 con el año 2019, los resultados indican que 13 son los cantones que han disminuido su participación. Estos son: Santa Cruz (9,17\%), Isabela (2,70\%), Alfredo Baquerizo Moreno (2,70\%), Salinas (2,52\%), San Cristóbal (2,46\%), Samborondón (2,43\%), El Chaco (2,24\%), Yantzaza (2,07\%), Huaquillas $(1,64 \%)$, La Libertad (1,49\%), La Concordia $(1,30 \%)$, Santa Elena $(0,56 \%)$, y Atahualpa $(0,24 \%)$.

Tabla 3.

Porcentaje de participación que disminuyó en alguna de las elecciones seccionales

\begin{tabular}{|l|l|l|l|l|}
\hline \multicolumn{1}{|c|}{ Provincia } & \multicolumn{1}{c|}{ Cantón } & \multicolumn{1}{c|}{$\mathbf{2 0 0 9}$} & \multicolumn{1}{c|}{$\mathbf{2 0 1 4}$} & \multicolumn{1}{c|}{$\mathbf{2 0 1 9}$} \\
\hline GUAYAS & PEDRO CARBO & 86,10 & 70,6 & 92,60 \\
\hline GUAYAS & LOMAS DE SARGENTILLO & 90,10 & 76,9 & 94,40 \\
\hline GUAYAS & SAN JACINTO DE YAGUACHI & 80,30 & 72,5 & 89,17 \\
\hline NAPO & CARLOS JULIO AROSEMENA TOLA & 86,70 & 78,5 & 90,81 \\
\hline LOS RIOS & QUINSALOMA & 85,40 & 84,3 & 92,24 \\
\hline EL ORO & ATAHUALPA & 81,30 & 89 & 88,76 \\
\hline SANTA ELENA & SANTA ELENA & 89,80 & 93,8 & 93,24 \\
\hline
\end{tabular}




\begin{tabular}{|l|l|l|l|l|}
\hline $\begin{array}{l}\text { SANTO DO- } \\
\text { MINGO DE LOS } \\
\text { TSACHILAS }\end{array}$ & LA CONCORDIA & 89,10 & 90,6 & 89,30 \\
\hline SANTA ELENA & LA LIBERTAD & 87,70 & 91,5 & 90,01 \\
\hline EL ORO & HUAQUILLAS & 83,50 & 87,9 & 86,26 \\
\hline $\begin{array}{l}\text { ZAMORA CHIN- } \\
\text { CHIPE }\end{array}$ & YANTZAZA & 70,40 & 81,3 & 79,23 \\
\hline NAPO & EL CHACO & 84,90 & 91,5 & 89,26 \\
\hline GUAYAS & SAMBORONDON & 83,10 & 87 & 84,57 \\
\hline GALAPAGOS & SAN CRISTOBAL & 72,30 & 76,2 & 73,74 \\
\hline SANTA ELENA & SALINAS & 87,10 & 91 & 88,48 \\
\hline GUAYAS & ALFREDO BAQUERIZO MORENO & 85,10 & 88,8 & 86,10 \\
\hline GALAPAGOS & ISABELA & 86,90 & 87,7 & 85,00 \\
\hline GALAPAGOS & SANTA CRUZ & 80,10 & 81,2 & 72,03 \\
\hline
\end{tabular}

Fuente: Consejo Nacional Electoral (CNE). Elaboración propia.

Como se puede observar en la tabla 4, los cinco de los 221 cantones con mayor participación entre el año 2009 en comparación con 2019, pertenecen a las regiones Costa y Amazonía. A continuación se describe la diferencia entre los periodos mencionados: Taisha (22,42\%), Jipijapa (16,77\%), Balzar (16,74\%), Aguarico (16,30\%), y Eloy Alfaro (15,78\%).

En Taisha existen 18.437 habitantes. El 12,2\% de las personas mayores a 15 años no saben leer ni escribir y la edad promedio es 19 años. Respecto a la provincia de Morona Santiago: los grupos de edad entre 20 a 24 años ascendieron del 7,8\% en 2001, al 8,6\% en 2010; y el 39,3\% de las personas mayores de 10 años en los últimos 6 meses no utilizaron celular, internet ni computadora (INEC 2010).

\section{Tabla 4.}

Los cinco cantones con mayor participación electoral entre el 2009 y 2019 
LA PARTICIPACIÓN ELECTORAL DEL 2009, 2014 Y 2019 EN ECUADOR: DIAGNÓSTICO POR CANTONES EN UN PAÍS CON VOTO OBLIGATORIO

\begin{tabular}{|l|l|l|l|}
\hline \multicolumn{1}{|c|}{ Provincia } & \multicolumn{1}{|c|}{ Cantón } & $\mathbf{2 0 0 9}$ & $\mathbf{2 0 1 9}$ \\
\hline MORONA SANTIAGO & TAISHA & 58,10 & 80,52 \\
\hline MANABI & JIPIJAPA & 71,90 & 88,67 \\
\hline GUAYAS & BALZAR & 69,00 & 85,74 \\
\hline ORELLANA & AGUARICO & 68,00 & 84,30 \\
\hline ESMERALDAS & ELOY ALFARO & 63,00 & 78,78 \\
\hline
\end{tabular}

Fuente: Consejo Nacional Electoral (CNE). Elaboración propia.

Como se puede observar en la tabla 5, los cinco de los 221 cantones con mayor participación entre el año 2014 en comparación con 2019, pertenecen a las regiones Costa, Sierra y Amazonía. A continuación se describe la diferencia entre los periodos mencionados: Pedro Carbo (22\%), Lomas de Sargentillo (17,50\%), San Jacinto de Yaguachi (16,67 \%), Carlos Julio Arosemena Tola $(12,31 \%)$ y Pangua $(11,57 \%)$.

En Pedro Carbo existen 43.436 habitantes. El 16,5\% de las personas mayores a 15 años no saben leer ni escribir y la edad promedio es 28 años. Respecto a la provincia: los grupos de edad entre los 20 a 24 años descendieron al $10,2 \%$ en 2001 , al $8,8 \%$ en 2010 ; y el $28,6 \%$ de las personas mayores de 10 años en los últimos 6 meses no utilizaron celular, internet ni computadora (INEC 2010).

\section{Tabla 5.}

Los cinco cantones con mayor participación electoral entre el 2014 y el 2019

\begin{tabular}{|l|l|l|l|}
\hline \multicolumn{1}{|c|}{ Provincia } & \multicolumn{1}{c|}{ Cantón } & \multicolumn{1}{c|}{$\mathbf{2 0 1 4}$} & \multicolumn{1}{c|}{$\mathbf{2 0 1 9}$} \\
\hline GUAYAS & PEDRO CARBO & 70,6 & 92,60 \\
\hline GUAYAS & LOMAS DE SARGENTILLO & 76,9 & 94,40 \\
\hline GUAYAS & SAN JACINTO DE YAGUACHI & 72,5 & 89,17 \\
\hline NAPO & CARLOS JULIO AROSEMENA TOLA & 78,5 & 90,81 \\
\hline COTOPAXI & PANGUA & 76,2 & 87,77 \\
\hline
\end{tabular}

Fuente: Consejo Nacional Electoral (CNE). Elaboración propia. 
Como se observa en la tabla 6 , en ninguna de las 24 capitales provinciales del país ha existido una disminución en cuanto a la participación de las dos elecciones seccionales, de 2009 y 2019. Todas muestran un aumento entre el $1,44 \%$ al 9,61\%. Los tres cantones donde más ascendió fueron en Machala $(9,61 \%)$, Zamora $(9,42 \%)$ y Guaranda $(9,15 \%)$. Mientras que los tres lugares donde su ascenso fue de menor escala pertenecen a San Cristóbal $(1,44 \%)$, Santa Elena (3,44\%) y Babahoyo (4,81\%).

Si se analiza a las tres capitales provinciales con mayor porcentaje de participación electoral, se puede encontrar el siguiente contexto. Las personas mayores de 15 años que no saben leer ni escribir llegan al 3,1\% en Machala; al 4,1\% en Zamora y al $18 \%$ en Guaranda. Mientras que la edad promedio para cada cantón es de 29, 26 y 28 años, respectivamente (INEC, 2010). Por tanto, si se constata los mismos datos para las tres capitales con menor porcentaje entre los años equivalentes, 2009 y 2019, se encuentra lo siguiente. Las personas mayores de 15 años que no saben leer ni escribir llegan al 1,2 \% en San Cristóbal, al 5,5\% en Santa Elena y al 6,7\% en Babahoyo. Mientras que la edad promedio para cada cantón es de 29, 27 y 28 años, respectivamente (INEC 2010).

Para encontrar una de las causas que expliquen este ascenso en el porcentaje de participación electoral, podría ser útil generar correlaciones de variables y regresiones, una vez que se ejecute el censo 2020, posterior a ello, un estudio cualitativo en los casos específicos podría obtener hallazgos significativos que aumente el nivel de confianza en las inferencias.

Si se observa por regiones, y analizando el porcentaje más alto y el más bajo, se puede destacar que, de la Amazonía fueron Zamora (9,42\%) y Orellana $(5,06 \%)$. De la Costa, Machala $(9,61 \%)$ y Santa Elena $(3,44 \%)$. Y de la Sierra, Guaranda $(9,15 \%)$ e Ibarra $(5,84 \%)$. Como se entenderá, de Galápagos consta una capital.

\section{Tabla 6.}

Porcentaje de participación electoral en capitales provinciales entre el 2009 y el 2019 
LA PARTICIPACIÓN ELECTORAL DEL 2009, 2014 Y 2019 EN ECUADOR: DIAGNÓSTICO POR CANTONES EN UN PAÍS CON VOTO OBLIGATORIO

\begin{tabular}{|l|l|c|c|c|}
\hline \multicolumn{1}{|c|}{ Provincia } & \multicolumn{1}{c|}{ Cantón } & $\mathbf{2 0 0 9}$ & $\mathbf{2 0 1 9}$ & Diferencia \\
\hline EL ORO & MACHALA & 75,50 & 85,11 & 9,61 \\
\hline ZAMORA CHINCHIPE & ZAMORA & 72,30 & 81,72 & 9,42 \\
\hline BOLIVAR & GUARANDA & 77,00 & 86,15 & 9,15 \\
\hline MORONA SANTIAGO & MACAS & 70,70 & 79,52 & 8,82 \\
\hline CANAR & AZOGUES & 58,50 & 67,14 & 8,64 \\
\hline ESMERALDAS & ESMERALDAS & 70,30 & 78,45 & 8,15 \\
\hline SANTO DOMINGO DE & SANTO DOMINGO & 75,10 & 83,13 & 8,03 \\
\hline LOS TSACHILAS & RIOBAMBA & 73,90 & 81,92 & 8,02 \\
\hline CHIMBORAZO & LAGO AGRIO & 76,20 & 83,55 & 7,35 \\
\hline SUCUMBIOS & AMBATO & 74,70 & 81,76 & 7,06 \\
\hline TUNGURAHUA & TULCAN & 76,50 & 83,27 & 6,77 \\
\hline CARCHI & PORTOVIEJO & 78,40 & 85,02 & 6,62 \\
\hline MANABI & PUYO & 75,30 & 81,69 & 6,39 \\
\hline PASTAZA & LOJA & 75,30 & 81,64 & 6,34 \\
\hline LOJA & CUENCA & 70,60 & 76,75 & 6,15 \\
\hline AZUAY & TENA & 81,30 & 87,45 & 6,15 \\
\hline NAPO & LATACUNGA & 81,90 & 87,94 & 6,04 \\
\hline COTOPAXI & QUITO & 75,90 & 81,80 & 5,90 \\
\hline PICHINCHA & IBARRA & 78,60 & 84,44 & 5,84 \\
\hline IMBABURA & GUAYAQUIL & 72,50 & 79,58 & 5,08 \\
\hline GUAYAS & ORELLANA & 87,06 & 5,06 \\
\hline ORELLANA & BABAHOYO & 87,30 & 4,81 \\
\hline LOS RIOS & SANTA ELENA & 93,24 & 3,44 \\
\hline SANTA ELENA & SAN CRISTOBAL & 73,74 & 1,44 \\
\hline GALAPAGOS & & & \\
\hline
\end{tabular}

Fuente: Consejo Nacional Electoral (CNE). Elaboración propia.

Como se observa en la tabla 7 , en esta ocasión existen 13 capitales provinciales donde sí ha existido una disminución de la participación en las dos elecciones seccionales, de 2014 y 2019. Esas muestran un descenso entre el $-0,01 \%$ al $-2,46 \%$, siendo porcentajes pequeños. 
Los dos cantones donde más ascendieron fueron, nuevamente, Machala (2,21\%), y Cuenca (1,65\%). Mientras que los dos lugares donde su ascenso fue de menor escala pertenecen a Tena $(0,05 \%)$ y Riobamba $(0,32 \%)$. Del mismo modo, San Cristóbal $(-2,46 \%)$, Zamora (-1,18\%) y Guayaquil $(-1,12 \%)$ son las tres ciudades en donde la participación electoral mayormente decreció en el periodo analizado.

Por tanto, Machala es un cantón que aumenta su porcentaje de participación en las tres elecciones: 2009, 2014 y 2019. Mientras que San Cristóbal ocupa el último puesto en los tres sufragios. Un caso atípico es Zamora, dado que entre 2009 a 2014 se ubica dentro de las tres capitales provinciales con aumento en su participación, pero de 2014 a 2019 baja su porcentaje quedando entre los últimos lugares.

Respecto a Cuenca, como el segundo cantón con mayor participación, y Guayaquil, con el antepenúltimo puesto, se puede contextualizar lo siguiente. Las personas mayores de 15 años que no saben leer ni escribir llega al $4,9 \%$ en la capital del Azuay y al 3,1\% en la capital del Guayas. Mientras que la edad promedio para ambos lugares es de 29 años (INEC 2010).

En 2014, en Cuenca fue electo Hugo Cabrera con el 52,4\% de los votos. Su predecesor fue Paúl Granda y el sucesor en 2019 es Pedro Palacios. Mientras que en Guayaquil, Jaime Nebot ganó en 2014 con el 59,5\%, y la sucesora es Cynthia Viteri. En el primer caso, el candidato Cabrera perdió la reelección, y en el segundo, Nebot después de 19 años en el poder, no se postuló.

Si se observa por regiones, y analizando el porcentaje más alto y el más bajo, se puede destacar que de la Amazonía fueron Tena $(0,05 \%)$ y Zamora (-1,18\%). De la Costa, Machala (2,21\%) y Guayaquil $(-1,12 \%)$. Y de la Sierra, Cuenca (1,65\%) y Loja (-0,76\%). Como se entenderá, de Galápagos consta una capital.

\section{Tabla 7.}

Porcentaje de participación electoral en capitales provinciales entre el 2014 y el 2019 
LA PARTICIPACIÓN ELECTORAL DEL 2009, 2014 Y 2019 EN ECUADOR: DIAGNÓSTICO POR CANTONES EN UN PAÍS CON VOTO OBLIGATORIO

\begin{tabular}{|c|c|c|c|c|}
\hline Provincia & Cantón & 2009 & 2019 & Diferencia \\
\hline El oro & Machala & 82,9 & 85,11 & 2,21 \\
\hline Azuay & Cuenca & 75,1 & 76,75 & 1,65 \\
\hline Cañar & Azogues & 65,5 & 67,14 & 1,64 \\
\hline Bolivar & Guaranda & 85 & 86,15 & 1,15 \\
\hline Tungurahua & Ambato & 81 & 81,76 & 0,76 \\
\hline Cotopaxi & Latacunga & 87,3 & 87,94 & 0,64 \\
\hline $\begin{array}{l}\text { Santo domingo } \\
\text { de los tsachilas }\end{array}$ & Santo domingo & 82,6 & 83,13 & 0,53 \\
\hline Esmeraldas & Esmeraldas & 78 & 78,45 & 0,45 \\
\hline Imbabura & Ibarra & 84,1 & 84,44 & 0,34 \\
\hline Chimborazo & Riobamba & 81,6 & 81,92 & 0,32 \\
\hline Napo & Tena & 87,4 & 87,45 & 0,05 \\
\hline Pastaza & Puyo & 81,7 & 81,69 & $\overline{-}$ \\
\hline Manabi & Portoviejo & 85,1 & 85,02 & $\begin{array}{l}-\quad 0,08 \\
\end{array}$ \\
\hline Pichincha & Quito & 81,9 & 81,80 & $-\quad 0,10$ \\
\hline Orellana & Orellana & 87,5 & 87,06 & - $\quad 0,44$ \\
\hline Santa elena & Santa elena & 93,8 & 93,24 & 0,56 \\
\hline Carchi & Tulcan & 84 & 83,27 & 0,73 \\
\hline Loja & Loja & 82,4 & 81,64 & 0,76 \\
\hline Los rios & Babahoyo & 88 & 87,11 & 0,89 \\
\hline Morona santiago & Macas & 80,5 & 79,52 & - $\quad 0,98$ \\
\hline Sucumbios & Lago agrio & 84,6 & 83,55 & 1,05 \\
\hline Guayas & Guayaquil & 80,7 & 79,58 & $-\quad 1,12$ \\
\hline Zamora chinchipe & Zamora & 82,9 & 81,72 & 1,18 \\
\hline Galapagos & San cristobal & 76,2 & 73,74 & 2,46 \\
\hline
\end{tabular}

Fuente: Consejo Nacional Electoral (CNE). Elaboración propia.

\section{Reflexiones finales}

A continuación se presentarán las conclusiones. Para analizar una de las dimensiones de la calidad de la democracia se debe primero mapear algunos 
enfoques que proporciona la literatura sobre qué es la democracia. De tal forma se utilizó a Schumpeter (1975), Dahl (1991), O`Donnell (2010), Schmitter y Karl (1996), entre otros.

También se describió la calidad de la democracia, apoyado de los autores Munck y Verkuilen (2002), Barreda (2013), Altman y Pérez-Liñán (2002), Mainwaring y Pérez-Liñán (2008), Diamond y Morlino (2004), Levine y Molina (2011), entre otros. Asimismo, se han desarrollado diferencias en sus observaciones, algunos analizan los procedimientos y no sus resultados. Un tema, sin duda, con mucho debate de por medio.

Posteriormente, se analizaron algunos enfoques sobre la participación electoral que provienen desde la literatura, por ejemplo con autoras y autores como Wagner, Johann y Kritzinger (2012), Lagos (2018), Franklin, Marsh y Lyons (2004), Morales y Reveco (2018), Geissel y Jung (2018), Santana, Ramón y Rama (2019), Hadjar y Beck (2010), entre otros. Siendo este tema el eje central de la investigación, de la evidencia empírica y de la pregunta que guía todo el trabajo, bajo la siguiente interrogante ¿cuál es el diagnóstico descriptivo de la participación electoral en las votaciones municipales del 2009, 2014 y 2019 en el Ecuador?

Para concluir la parte del marco teórico se destaca que, al ser una investigación a nivel subnacional, se toma como referencia a Betancourt (2018), en donde examina la calidad de la democracia. Por tanto, el aporte radica en contribuir con insumos e información descriptiva actualizada, sobre la participación electoral, que servirá como elemento clave para futuros trabajos que busquen las causas que expliquen este comportamiento durante elecciones de 2009 a 2019 en 221 cantones, en un país donde el voto es obligatorio.

Respecto a la parte metodológica, se seleccionó una de las dimensiones que proponen Diamond y Morlino (2004). Tomando en cuenta la extensión del presente documento, la pertinencia y accesibilidad de datos, se escogió la participación electoral para las tres votaciones locales. Por tanto, se entrega a la comunidad académica, política, lideresas y líderes de la sociedad y público en general una investigación de corte descriptivo, que brinde elementos para 
conocer el diagnóstico en un país cuyo sufragio no es opcional. Se deja claro que existen otras formas metodológicas que pueden ser abordadas sobre la participación durante las votaciones, por ejemplo, analizar las configuraciones sociológicas, psicológicas o motivacionales (Hadjar y Beck, 2010: 521), no obstante, el objetivo del documento era generar un primer diagnóstico de 2009 al 2019 en el país.

En relación a los resultados, se destaca que la participación, aquí medida como el porcentaje de sufragantes respecto a electores, no es la única forma de observarla, y que, como otras podría ser debatible. Por ejemplo, es más complejo analizar para 221 lugares el acceso efectivo de las personas para acercarse a las urnas, especialmente en el sector rural, estudiar el sufragio por convicción en un país donde el voto es obligatorio, descubrir las formas en que se desenvuelven las relaciones entre electores y políticos con el clientelismo o el patronazgo, entendido más como una estrategia que utiliza la gente para resolver sus problemas y como modo de sobrevivencia (Auyero 2012: 17). Según Jiménez (2019) también se podría observar desde el contenido de los mensajes que promocionan las y los candidatos, al estar cercanos o alejados de las necesidades de la población, para ver las probabilidades en que las personas estarían más dispuestas en acudir a las urnas. Como se observa, existen múltiples formas de enfocarla, por tanto, se deja claro el alcance del trabajo.

La tendencia de participación varía entre el $60 \%$ al $90 \%$ en las tres elecciones seccionales: 2009, 2014 y 2019. El cantón Déleg ocupa el último puesto, donde 4 de cada 10 personas fueron a votar. Mientras que Isidro Ayora tiene el primer lugar con 9 de cada 10 ciudadanos. En 18 de los 221 cantones, el porcentaje disminuyó en una de las tres elecciones seccionales. Ninguno de ellos pertenece a la Sierra. Los tres cantones con mayor participación, y una diferencia entre 2009 y 2019, pertenecieron a Taisha (22,42\%), Jipijapa $(16,77 \%)$ y Balzar (16,74\%). Del mismo modo, entre el 2014 y el 2019, fueron Carbo (22\%), Sargentillo (17,50\%) y Yaguachi (16,67\%).

Respecto a las capitales provinciales, no existe ninguna disminución entre 2009 y 2019. Los tres cantones donde más ascendió la participación fueron Machala $(9,61 \%)$, Zamora $(9,42 \%)$ y Guaranda $(9,15 \%)$. Mientras 
que, donde su ascenso fue menor, pertenecen a San Cristóbal (1,44\%), Santa Elena $(3,44 \%)$ y Babahoyo (4,81\%).

Del mismo modo, existen 13 capitales que disminuyeron la participación entre las elecciones de 2014 y 2019. Los dos cantones donde más ascendió fueron Machala $(2,21 \%)$ y Cuenca $(1,65 \%)$. Mientras que San Cristóbal (-2,46\%), Zamora (-1,18\%) y Guayaquil (-1,12\%) fueron las que más decrecieron. Todos los descensos varían entre $-0,01 \%$ a $-2,46 \%$, siendo porcentajes pequeños.

Esta investigación deja nuevas interrogantes ¿Qué explica que entre las votaciones de 2014 a 2019 la participación disminuyó, aunque en porcentajes pequeños, en algunas capitales provinciales de un país donde el voto es obligatorio? ¿Qué factores revelan la repetición en Déleg e Isidro Ayora como los cantones con menor y mayor participación para las tres elecciones? ¿Qué ocurre en el cantón Machala para que sea la capital provincial con mayor participación en el comparativo 2009-2019 y 2014-2019? 
LA PARTICIPACIÓN ELECTORAL DEL 2009, 2014 Y 2019 EN ECUADOR: DIAGNÓSTICO POR CANTONES EN UN PAÍS CON VOTO OBLIGATORIO

\section{Bibliografía}

Acemoglu, Daron y James Robinson (2012). Por qué fracasan los países: los orígenes del poder, la prosperidad y la pobreza. Barcelona: Centro Libros PAPF, Deusto.

Altman, David y Aníbal Pérez-Linán (2002). “Assessing the Quality of Democracy: Freedom, Competitiveness and Participation in Eighteen Latin American Countries”. En Democratization 9, 2: págs. 85-100

Auyero, Javier (2012). "Los sinuosos caminos de la etnografía política”. En Pleyade 10: págs. 15-36

Barreda, Mikel (2013). "La calidad de las democracias latinoamericanas: medición y claves explicativas". En La calidad de la democracia: perspectivas desde América Latina, editado por Mantilla, Sebastián y Gerardo Munck: págs. 155-185. Quito: CELAEP

Betancourt, Edgar (2018). Medición de la calidad de la democracia en Quito. Tesis de FLACSO Ecuador.

Castiglioni, Rossana y Cristóbal Rovira (2016). "Challenges to Political Representation in Contemporary Chile". En Journal of Politics in Latin America 8, 3: págs. 3-24

Consejo Nacional Electoral (2014). "Indicadores electorales". Visita de 15 de julio de 2019. Disponible en www.cne.gob.ec/es/estadisticas/ publicaciones/category/136-atlas-electoral-2009-2014.

Coppedge, Michael (2012). Democratization and Research Methods. New York: Cambridge University Press.

Dahl, Robert (1991). "La democracia y sus críticos". En La segunda transformación democrática: de la ciudad-estado al estado nacional: 
págs. 257-270. Buenos Aires: Paidós.

Dalton, Russell (2009). The Good Citizens. How a Younger Generation is Reshaping American Politics. Washington D.C: CQ Press.

Della Porta, Donatella (2013). “Análisis comparativo: la investigación basada en casos frente a la investigación basada en variables". En Della Porta, Donatella y Michael Keating (eds.). Enfoques y metodologías de las ciencias sociales. Una perspectiva pluralista, págs. 211-236. Madrid: Akal.

Diamond, Larry y Leonardo Morlino (2004). "The Quality of Democracy. An Overview”. En Journal of Democracy 15, 4: págs. 20-31

Franklin, Mark, Michael Marsh y Patrick Lyons (2004). "Generational basis of turnout decline in established democracies". En Acta Politica 39, 2: págs. 115-151. doi: 10.1057/palgrave.ap.5500060

Geissel, Brigitte y Stefan Jung (2018). "Recall in Germany: explaining the use of a local democratic innovation". En Democratization 25, 8: págs. 1358-1378. doi: 10.1080/13510347.2017.1398735

Giraudy, Agustina, Eduardo Moncada y Richard Snyder (2019). "Empirical and Theoretical Frontiers of Subnational Research in Comparative Politics". En Inside Countries: Subnational Research in Comparative Politics: págs. 353-367. doi:10.1017/9781108678384.011

Hadjar, Andreas y Michael Beck (2010). "Who does not participate in elections in Europe and why is this?” En European Societies 12, 4: págs. 521-542. doi: 10.1080/14616696.2010.483007

Hernández Roberto, Carlos Fernández y María del Pilar Baptista (2010). Metodología de la investigación. México: McGraw-Hill/Interamericana Editores. 
LA PARTICIPACIÓN ELECTORAL DEL 2009, 2014 Y 2019 EN ECUADOR: DIAGNÓSTICO POR CANTONES EN UN PAÍS CON VOTO OBLIGATORIO

INEC (2010). Instituto Nacional de Estadística y Censos. Censo de población y vivienda. Ecuador.

Jiménez, Eduardo (2019). “¿En el Ecuador del 2019, las propuestas de candidatos municipales representan las demandas ciudadanas, o son ofrecimientos que no dicen nada?”. En Revista de Ciencias Políticas y Relaciones Internacionales 8: págs. 40-72.

Lagos, Marta (2018). El fin de la tercera ola de democracias. Chile: Corporación Latinobarómetro.

LAPOP (2018). Cultura política de la democracia en Ecuador y en las Américas, 2016/17: Un estudio comparado sobre democracia y gobernabilidad. Estados Unidos: Vanderbilt-Barómetro de las AméricasGrupo 50+1-UDLA.

Levine, Daniel y José Enrique Molina (2011). "Calidad de la democracia: fortalezas y debilidades en América Latina". Revista Latinoamericana de Política Comparada 05: págs. 95-123.

Luna, Juan Pablo (2008). "Partidos políticos y sociedad en Chile. Trayectoria histórica y mutaciones recientes". En Fontaine, Arturo; Cristián Larroulet; Jorge Navarrete y Ignacio Walker (eds.). Reforma de los partidos politicos en Chile. Santiago: PNUD, CEP, Libertad y Desarrollo, Proyectamérica y CIEPLAN.

Mainwaring, Scott y Aníbal Pérez-Liñán (2008). "Regime legacies and democratization: explaining variance in the level of democracy in Latin America, 1978-2004". En Working Paper Kellogg Institute for International Studies 354: págs. 1-31

McKay, Lawrence (2019). "'Left behind' people, or places? The role of local economies in perceived community representation". En Electoral Studies 60: págs. 1-11 
Morales, Mauricio y Bastián Reveco (2018). "El efecto de las generaciones políticas sobre la participación electoral. El caso de Chile, 1999-2013" En Perfiles latinoamericanos 26, 52: págs. 1-27

Morlino, Leonardo (2013). La calidad de las democracias en América Latina: págs. 36-102. Maryland: IDEA-LUISS.

Munck, Gerardo y Jay Verkuilen (2002). "Conceptualizando y midiendo la democracia: una evaluación de índices alternativos". En Política y Gobierno, IX, 2: págs. 403-441

O`Donnell, Guillermo (1992). “¿Democracia delegativa?”. En Cuadernos del CLAEH 17, 1: págs. 6-20

(2010). "Democracia, agencia y estado": págs. 23-45. Buenos Aires: Prometeo.

(2011). "Nuevas reflexiones acerca de la democracia delegativa (DD)”. En Democracia Delegativa, editado por O’Donnell, Guillermo, Osvaldo Iazzetta y Hugo Quiroga: págs. 19-33. Buenos Aires: Prometeo

Programa de las Naciones Unidas para el Desarrollo - PNUD (2004). "La democracia en América Latina". En Indicadores de desarrollo de la democracia, págs. 73-135; Nota técnica sobre el índice electoral: págs. 213-228. PNUD: New York.

Ragin, Charles (2007). "El uso de los métodos comparativos para estudiar la diversidad". En La construcción de la investigación social, págs: 177212. Bogotá: Siglo del Hombre Editores/SAGE.

Santana, Andrés, José Ramón Montero y José Rama (2019). “Las elecciones parlamentarias chilenas de 2017: características de los votantes y factores de voto”. En América Latina Hoy 81: págs. 147-171 
LA PARTICIPACIÓN ELECTORAL DEL 2009, 2014 Y 2019 EN ECUADOR: DIAGNÓSTICO POR CANTONES EN UN PAÍS CON VOTO OBLIGATORIO

Scharpf, Fritz (1999). Governing in Europe: Effective and Democratic? Oxford: Oxford University Press.

Schmitter, Philippe y Terry Lynn Karl (1996). "Qué es... y qué no es democracia". En El resurgimiento global de la democracia, editado por Diamond, Larry y Mark F. Plattner: págs. 37-49. México: UNAM

Schumpeter, Joseph (1975). Capitalism, Socialism and Democracy. New York: Harper and Row.

Sen, Amartya (2001). "Las teorías del desarrollo en el siglo XXI". En Revista Leviatán 84: págs. 65-84.

Wagner, Markus, David Johann y Sylvia Kritzinger (2012). "Voting at 16: Turnout and the quality of vote choice". En Electoral Studies 31, 2: págs. 372-383. doi: 10.1016/j.electstud.2012.01.007 


\section{ANEXOS}

\section{Anexo 1.}

Bases de datos sobre la democracia: una evaluación

\begin{tabular}{|c|c|c|}
\hline Nombres & Fuerzas & Debilidades \\
\hline \multirow{4}{*}{$\begin{array}{l}\text { ACLP: Álvarez } \\
\text { Cheibub, Limon- } \\
\text { gi y Przeworski }\end{array}$} & Identificación de atributos: cargos. & \multirow{4}{*}{$\begin{array}{l}\text { Definición minimalista: omisión } \\
\text { de la participación y el poder de } \\
\text { fijar la agenda. }\end{array}$} \\
\hline & Lógica conceptual. & \\
\hline & Selección adecuada de indicadores. & \\
\hline & $\begin{array}{l}\text { Reglas de mediciones claras y } \\
\text { detalladas. }\end{array}$ & \\
\hline Arat & $\begin{array}{l}\text { Identificación de atributos: cargos } \\
\text { y poder de fijar la agenda. }\end{array}$ & $\begin{array}{l}\text { Lógica conceptual: problema de } \\
\text { superposición (conflation) }\end{array}$ \\
\hline \multirow[b]{2}{*}{ Bollen } & \multirow{2}{*}{$\begin{array}{l}\text { Identificación de atributos: cargos, } \\
\text { poder de fijar la agenda e impar- } \\
\text { cialidad. }\end{array}$} & $\begin{array}{l}\text { Definición minimalista: omisión } \\
\text { de la participación. }\end{array}$ \\
\hline & & $\begin{array}{l}\text { Lógica conceptual: problema de } \\
\text { superposición. Alcance empírico } \\
\text { (temporal) restringido }\end{array}$ \\
\hline \multirow{3}{*}{$\begin{array}{l}\text { Coppedge y } \\
\text { Reinicke }\end{array}$} & $\begin{array}{l}\text { Identificación de atributos: impar- } \\
\text { cialidad. }\end{array}$ & $\begin{array}{l}\text { Definición minimalista: omisión } \\
\text { de la participación, los cargos y el } \\
\text { poder de fijar la agenda. }\end{array}$ \\
\hline & $\begin{array}{l}\text { Prueba de confiabilidad entre } \\
\text { analistas. }\end{array}$ & $\begin{array}{l}\text { Alcance empírico (temporal) } \\
\text { restringido. }\end{array}$ \\
\hline & $\begin{array}{l}\text { Procedimiento de agregación } \\
\text { complejo. }\end{array}$ & \\
\hline \multirow{4}{*}{ Freedom House } & \multirow{4}{*}{$\begin{array}{l}\text { Alcance empírico (espacial) } \\
\text { amplio. }\end{array}$} & Definición maximalista. \\
\hline & & $\begin{array}{l}\text { Lógica conceptual: problema de } \\
\text { superposición. }\end{array}$ \\
\hline & & Múltiples problemas de medición. \\
\hline & & $\begin{array}{l}\text { Procedimiento de agregación } \\
\text { inadecuado. }\end{array}$ \\
\hline \multirow{4}{*}{ Polity IV } & $\begin{array}{l}\text { Identificación de atributos: cargos } \\
\text { y poder de fijar la agenda. }\end{array}$ & $\begin{array}{l}\text { Definición minimalista: omisión } \\
\text { de la participación. }\end{array}$ \\
\hline & $\begin{array}{l}\text { Reglas de mediciones claras y } \\
\text { detalladas. }\end{array}$ & $\begin{array}{l}\text { Lógica conceptual: problema de } \\
\text { redundancia. }\end{array}$ \\
\hline & $\begin{array}{l}\text { Pruebas de confiabilidad entre } \\
\text { analistas. }\end{array}$ & $\begin{array}{l}\text { Procedimiento de agregación } \\
\text { inadecuado. }\end{array}$ \\
\hline & Alcance empírico amplio. & \\
\hline \multirow{3}{*}{ Vanhanen } & Reglas de medición claras. & $\begin{array}{l}\text { Definición minimalista: omisión } \\
\text { de cargos y el poder de fijar la } \\
\text { agenda. }\end{array}$ \\
\hline & Alcance empírico amplio. & Indicadores cuestionables. \\
\hline & Replicabilidad. & $\begin{array}{l}\text { Procedimientos de agregación } \\
\text { inadecuada. }\end{array}$ \\
\hline
\end{tabular}

Fuente: Munck y Verkuilen, 2002: 434. 


\section{Anexo 2.}

La participación electoral 1990-2002 en América Latina

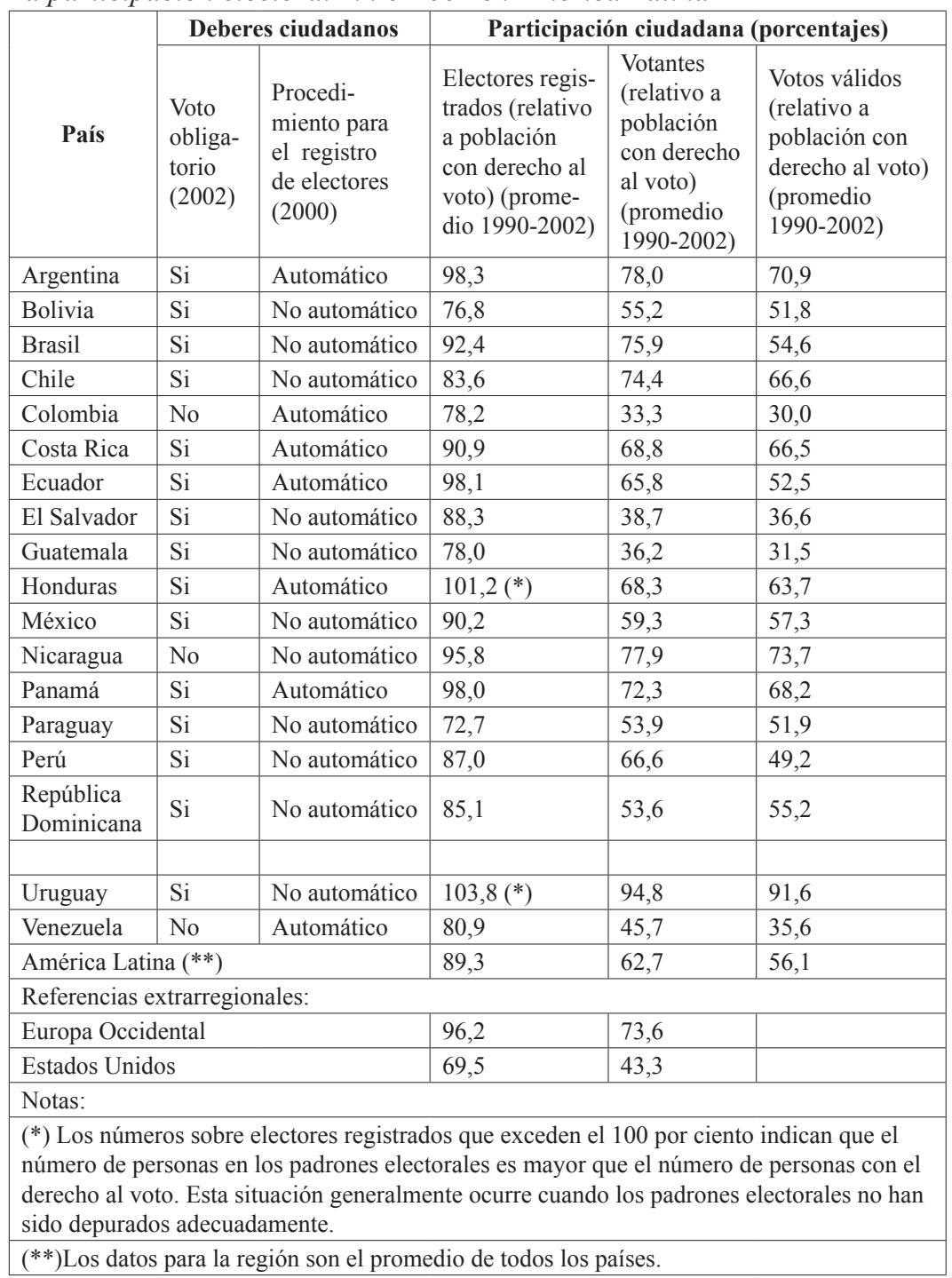

Fuente: Programa de las Naciones Unidas para el Desarrollo (PNUD), 2004: 87. 